\title{
Tourist route optimization design and algorithm
}

\author{
Xu Yong-lin ${ }^{1}$, Zhang Sheng-zhi ${ }^{2}$, Yang Jin $^{3}$ \\ (1Mathematics and Computer College, Northwestern Minorities \\ University, Lanzhou, Gansu,730030,China. \\ 2Department of Mathematics, Gansu Normal University for Nationalities, \\ Hezuo, Gansu, 747000, China. \\ 3The major of Higher Education in the College of Education of Lanzhou \\ University, Lanzhou, Gansu,730030,China.) \\ E-mail: xuyonglin000@163.com
}

\begin{abstract}
. Abstract
Take ten tourist attractions in Lanzhou as the example to design one-day trip at Lanzhou plan model. Through Dijskra algorithm to find the shortest path and the path which spent the shortest time, and analys the result to get the way which is the best solution, use the target node in the first time as the source point, calculate the optimal path of the next goal node, iterate them one by one according to their own needs, and then optimize the tourism of the shortest path.

Key word: Dijskra algorithm, Shortest path, Tourism, Model
\end{abstract}

\section{Introduction}

Dijskra algorithm is a typical shortest path algorithm, is also a representative shortest path algorithm. There are usually two kinds of expression: one is using a kind of permanent label or temporary label to describe it, another is to use the OPEN and CLOSE table to express. Generating the algorithm is according to the order of the increasing of the length of path:Vertexes set $\mathrm{V}$ will be divided into two groups: one group is a collection of vertices S. only containing Source point $\mathrm{V}_{0}$ at first ; Another group is the set of the points that is not determined: $\mathrm{V}-\mathrm{S}=\mathrm{T}$. The points in the set of containing undetermined points will be put in the set of Source point $S$ the order of the increasing.

(1)From where $V_{0}$ to $T$ any one vertex in the shortest path length, than from the source point where $V_{0}$ to $S$ in other length of each vertex;(2)Each distance value corresponding to a vertex.From where $V_{0}$ to the vertex is the length of the $\mathrm{S}$ in, from where $\mathrm{V}_{0}$ to the vertex include $\mathrm{S}$ only for intermediate vertex of the shortest path length is $T$. Can prove where $V_{0}$ to $T$ vertex vitamin $V_{k}$, where $V_{0}$ to vitamin $\mathrm{V}_{\mathrm{k}}$ in the direct path of weights, and from where $\mathrm{V}_{0} \mathrm{~S}$ vertex to the path of vitamin $\mathrm{V}_{\mathrm{k}}$ in the sum of weights.

Algorithm steps as shown below

Step 1: initialization, make $S$ for source point where $V_{0}$, $T$ for other vertices, $\mathrm{T}$ in the vertex value corresponding to the distance;Step 2: pick up a its distance 
from the $\mathrm{T}$ value is the smallest vertex $\mathrm{W}$ (and not in the $\mathrm{S}$ ), put in S;Step 3: Changes in the rest of the $\mathrm{T}$ vertex distance value: $\mathrm{f}$ will be added to $\mathrm{W}$ intermediate vertex, shorten the distance from where $V_{0}$ to $V_{i}$ value, modify the rest $\mathrm{T}$ vertex in the distance.Repeat the above steps 2,3 , until all the vertices in the $\mathrm{S}, \mathrm{W}$ and $\mathrm{V}_{\mathrm{i}}$ is equal.

\section{Model assumes}

(1) Assume that take the bus to downtown Lanzhou students get off at the provincial government;

(2) Assuming that students in Lanzhou play only bus and walk for two kinds of transportation;

(3) The figure of the intersection as a node;

(4) Hypothesis of normal adults walking speed of $1 \mathrm{~m} / \mathrm{s}$;

(5) Assumes that the speed of the bus under the conditions of roads free of 12 $\mathrm{m} / \mathrm{s}$, under the busy road speed of $4 \mathrm{~m} / \mathrm{s}$;

6) Assumes that people at the bus stop waiting for the bus and get on an average time of $5 \mathrm{~min}$, the $300 \mathrm{~s}$;

(7) Take the scenic spot nearest the spots should choose the path of the node;

(8) Assuming that the bus can stop anywhere.

\section{The main symbols and variables}

$i$-Starting node label; $j$-End node label; $L_{i j}$-Between the actual path from the start node to the end node (non linear distance) ; $T_{i j}$ _ From the start node to the end node between the time of consumption;

$\mathrm{a}[\mathrm{i}][\mathrm{j}]$ — Right side (i,j); dist[i]—_ The current from the source to the vertex I special shortest path length;prev[i]__ From the source to the vertices of the shortest path I I front a vertex.

\section{The establishment and solving of the model}

Setting up and solving the model. With some travel agency website, available Lanzhou city tourist attractions rankings are shown in table 1 below.

Table1. Lanzhou city tourist attractions ranked ranking

1

2

3

4

5

6

7

8

9

10

11

12
Scenic spot name

Gansu province museum ( Lcon K )

The mountain forest park

BeiLin in lanzhou

XingLong mountain nature reserve

The Yellow River mother sculpture ( Lcon J)

LuTuSi government

DaZhong lane ( Lcon C)

Joy west garden

The Yellow River railway bridge

LanShan park

XiGuan mosque ( Lcon K )

BaiYunGuan temple ( Lcon G) 


$\begin{array}{lc}13 & \text { Earthquake museum } \\ 14 & \text { LanZhou chenghuang temple ( Lcon B) } \\ 15 & \text { AnNing Botanical garden } \\ 16 & \text { Waterwheel museum park ( Lcon H) } \\ 17 & \text { Small west lake ( Lcon I) } \\ 18 & \text { BaiTa mountain park ( Lcon E) } \\ 19 & \text { WuQuan mountain park } \\ 20 & \text { ZhongShan bridge ( Lcon D) }\end{array}$

In table 1, because a day's time is limited, so only choose the address in the Chengguan district, Qilihe district scenic spot. Using the world's most advanced satellite cloud pictures - Google's satellite map images of Lanzhou Chengguan and Qilihe district map as shown in figure 1 (annex 1), on the basis of figure 1 by PhotoShop software to each scenic spot (B-K), each intersection (node 1-23) and the bus, walking can involve the identity, the road of as shown in figure 2 (annex 1), using Google's satellite map of distance measurement tools, roughly measure the distance between two adjacent nodes. In order to make the measured value has strong persuasive and accuracy, and repeated measurements 3 times for each section, the finally take the average of 3 times as the final measured values, the section of measuring the results are shown in table 2 as follows:

Table 2. The distance between two adjacent nodes

$\begin{array}{cccccc}\begin{array}{c}\text { Source } \\ \text { point }\end{array} & \begin{array}{c}\text { Purpose } \\ \text { node }\end{array} & \begin{array}{c}\text { The measured } \\ \text { value of the first }\end{array} & \begin{array}{c}\text { The } \\ \text { measured } \\ \text { value of the } \\ \text { second }\end{array} & \begin{array}{c}\text { The measured } \\ \text { value of the third } \\ \text { time }\end{array} & \begin{array}{c}\text { The } \\ \text { average }\end{array} \\ 1 & 2 & 448.020 & 447.500 & 449.100 & 448 \\ 2 & 3 & 251.048 & 252.050 & 250.472 & 251 \\ 3 & 4 & 205.007 & 204.992 & 206.125 & 205 \\ 4 & 5 & 229.678 & 227.978 & 228.673 & 229 \\ 4 & 23 & 262.571 & 264.672 & 263.185 & 263 \\ 5 & 6 & 283.567 & 285.800 & 284.023 & 284 \\ 6 & 7 & 285.010 & 287.200 & 386.375 & 386 \\ 7 & 8 & 402.478 & 400.378 & 401.207 & 401 \\ 7 & 9 & 347.789 & 348.056 & 346.350 & 347 \\ 9 & 11 & 339.449 & 342.618 & 340.005 & 340 \\ 9 & 10 & 340.816 & 342.809 & 338.140 & 340 \\ 10 & 11 & 257.380 & 255.787 & 256.592 & 257 \\ 8 & 12 & 555.387 & 554.379 & 555.290 & 555 \\ 12 & 13 & 153.289 & 154.739 & 152.592 & 153 \\ 13 & 14 & 320.381 & 321.334 & 319.394 & 320 \\ 10 & 14 & 237.299 & 239.689 & 238.890 & 239 \\ 13 & 16 & 660.128 & 662.144 & 658.279 & 660 \\ 16 & 15 & 217.788 & 218.489 & 216.479 & 217 \\ 15 & 14 & 558.512 & 559.683 & 559.479 & 559 \\ 16 & 17 & 1011.290 & 1010.956 & 1010.989 & 1011 \\ 17 & 19 & 454.463 & 453.890 & 455.479 & 454 \\ 15 & 18 & 943.629 & 944.479 & 944.790 & 944\end{array}$




$\begin{array}{llllll}17 & 19 & 777.890 & 777.083 & 777.283 & 777 \\ 19 & 20 & 209.676 & 210.389 & 210.490 & 210 \\ 20 & 22 & 481.387 & 479.790 & 480.483 & 480 \\ 18 & 22 & 854.482 & 855.200 & 853.088 & 854 \\ 21 & 22 & 438.589 & 438.012 & 437.749 & 438 \\ 1 & 5 & 294.756 & 295.890 & 295.005 & 295\end{array}$

Need to explain here that can be terminated start node, end node can also be a starting node, that is, the whole network diagram is an undirected graph. The same section has the following relationship: $L_{i j}=L_{j i}$

Model design.According to monophyletic shortest path problem, through the Dijskra algorithm design

computer program. Dijskra algorithm is monophyletic solution greedy algorithm of the shortest path problem. First set vertex set $\mathrm{S}$ and continuously make greedy choice to expand the collection. The initial, $\mathrm{S}$ only contains the source. uis a vertex of $G$, from the source to the only after $S$ peak road in between $u$ and become a special path from the source to $\mathrm{u}$, and current each node dist record an array of vertices $\mathrm{u}$, will $\mathrm{u}$ added to the $\mathrm{S}$, at the same time to make the necessary changes to your array dist. Once $\mathrm{S}$ contains all the vertices in $\mathrm{V}$, dist is recorded from the source to the length of the shortest path between all the other vertices. A vertex $\mathrm{V}$ is the source, and $\mathrm{a}$ is a two-dimensional array, a[i][j] said edge (i,j). When $(i, j) \notin E$, a[i][j] is a huge number, we use Float. MAX_VALUE to say it. dist[i] said that the current from the source to the vertex i special shortest path length. But this is calculated from the length of the shortest path between source vertex to the other, to find out the corresponding shortest path, using an array prev record the information of the shortest path. Array prev[i] records from the source to the vertices of the shortest path i I front a vertex. Initially, for all, $\operatorname{prev}[\mathrm{i}]=\mathrm{v}$. In Dijskra algorithm updates the shortest path length, as long as when $\operatorname{dist}[u]+a[u][i]<\operatorname{dist}[i]$,placing $\operatorname{prev}[\mathrm{i}]=\mathrm{u}$. When terminated Dijskra algorithm, can according to the array prev to find the shortest path from the source to the i before each vertex of a vertex, so as to find the shortest path from the source to the $i$.

solution of the model. Algorithm for the model, the use of Java language programming, programming

environment is JCreator LE.The operation of the procedures the results as follows:

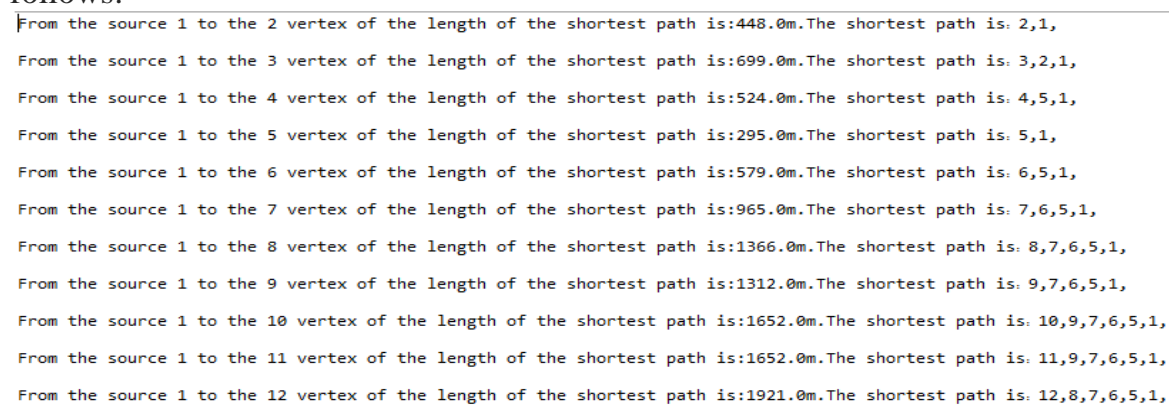




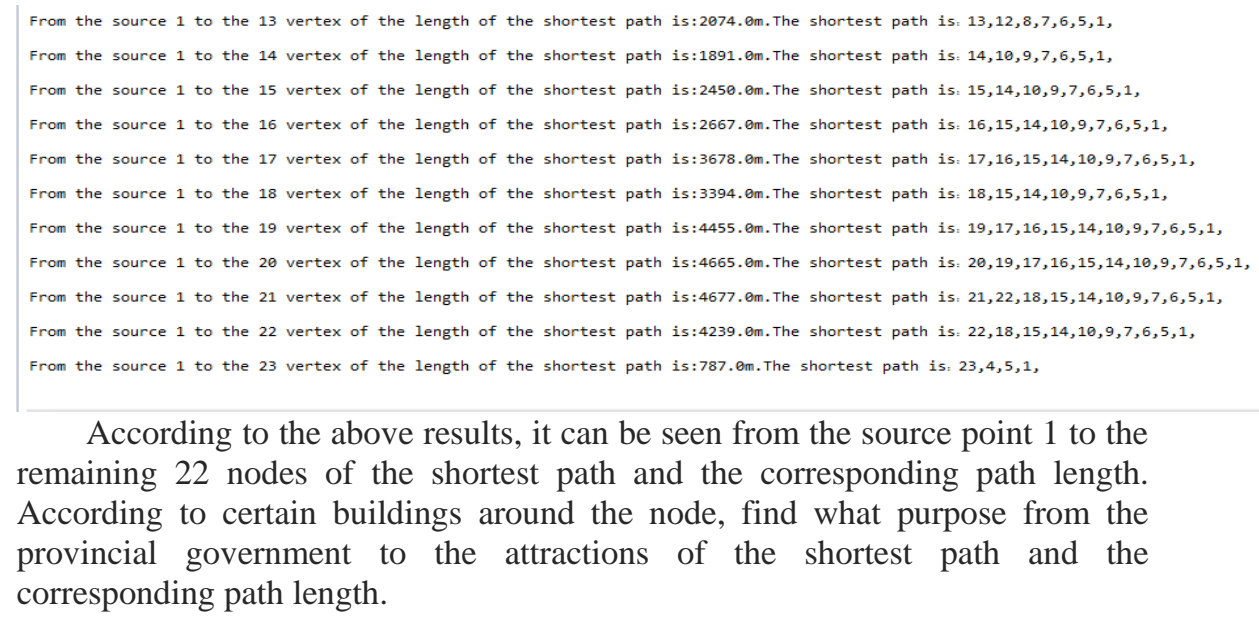

According to the above results, it can be seen from the source point 1 to the remaining 22 nodes of the shortest path and the corresponding path length. According to certain buildings around the node, find what purpose from the provincial government to the attractions of the shortest path and the corresponding path length.

\section{The establishment and solving the model}

Problem analysis.In model assumption, presupposing assuming normal adults walking speed of $1 \mathrm{~m} / \mathrm{s}$, the speed of the bus in the free state of $12 \mathrm{~m} / \mathrm{s}$, under the busy road speed of $4 \mathrm{~m} / \mathrm{s}$ and the people at the bus stop waiting for the bus and get on an average time of $5 \mathrm{~min}$, the $300 \mathrm{~s}$; Set $\mathrm{x}$ as the distance between any two nodes, then according to the above data equation can be divided into the following two conditions:

(1) When the road is free: $\quad x / 12+5 * 60>=x / 1$, Solution: $x<=327$

(2) When the road is busy: $\quad x / 4+5 * 60>=x / 1$, Solution: $x<=400$

The following conclusions: (1)when the road is in the idle state: when the distance between two nodes is less than $327 \mathrm{~m}$, walk more save time; When the distance between two nodes is larger than $327 \mathrm{~m}$, choose the bus more save time; When the distance between two nodes is equal to $327 \mathrm{~m}$, walk and ride the bus, it takes both at the same time;(2)Road in the busy state: when the distance between two nodes is less than $400 \mathrm{~m}$, walk more save time; When the distance between two nodes is larger than $400 \mathrm{~m}$, choose the bus more save time; When the distance between two nodes is equal to $400 \mathrm{~m}$, walk and ride the bus, it takes both at the same time. In the free state, the shortest path problem is the shortest time. According to the above conclusion and table 2, measured between two adjacent nodes used in the schedule, such as table3:

Table 3.The schedule between two adjacent nodes

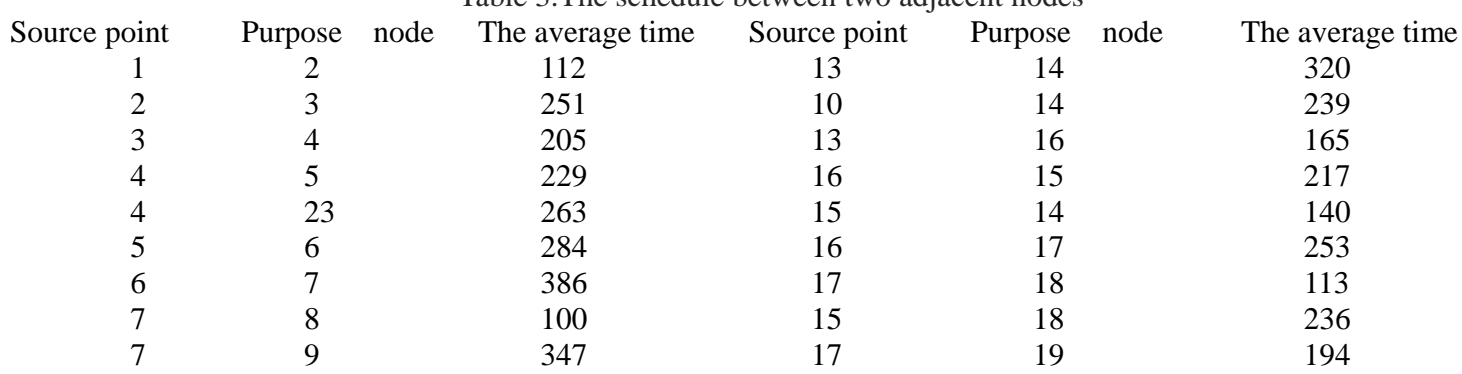




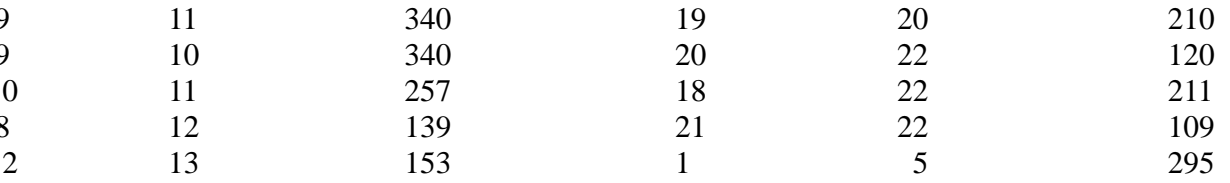

Which can be terminated starting node and end node can also be a starting node, that is, the whole network diagram is an undirected graph. The same section has the following relationship:

$$
T_{i j}=T_{j i}
$$

Model design. This is required by the time the least path. In question one, is due to the requirement of monophyletic shortest path, so use the Dijskra algorithm. Will change the length of each stage in the time spent on each of the sections, reoccupy Dijskra algorithm can work out the path of the time spent at least. Get on the path through the various sections of the time it takes to add up, and can be obtained from the source to the minimum amount of time spent at various points in the figure.

According to the previous design ideas, to still use Dijskra algorithm to solve the problem. In specific computer program, the original records each section length of the value in a two-dimensional array can be replaced with the data in table 3 , the rest of the code remains the same in the program.

Solution of the model. Algorithm for the model, the use of Java language programming, programming

environment is JCreator LE.The operation of the procedures the results as follows:

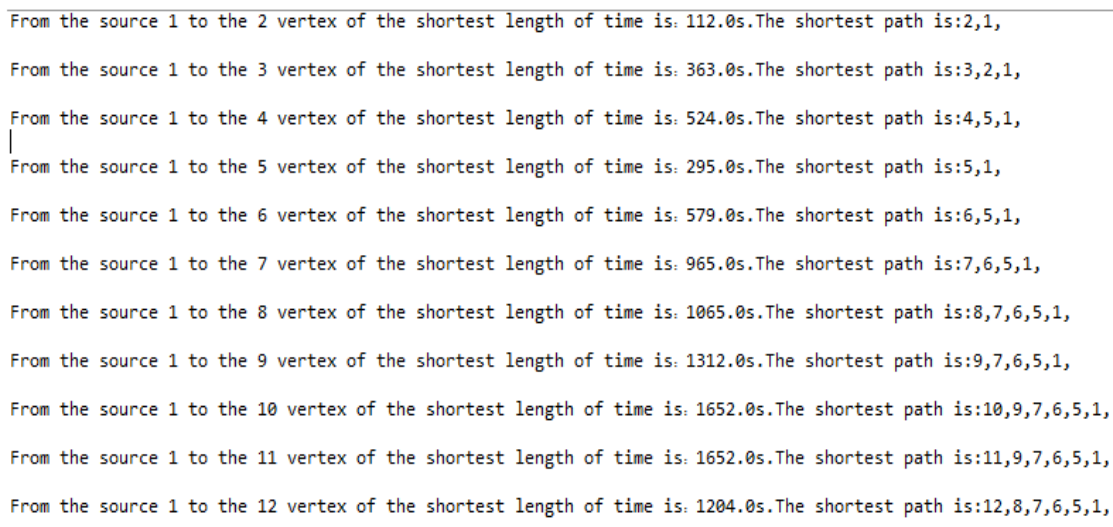




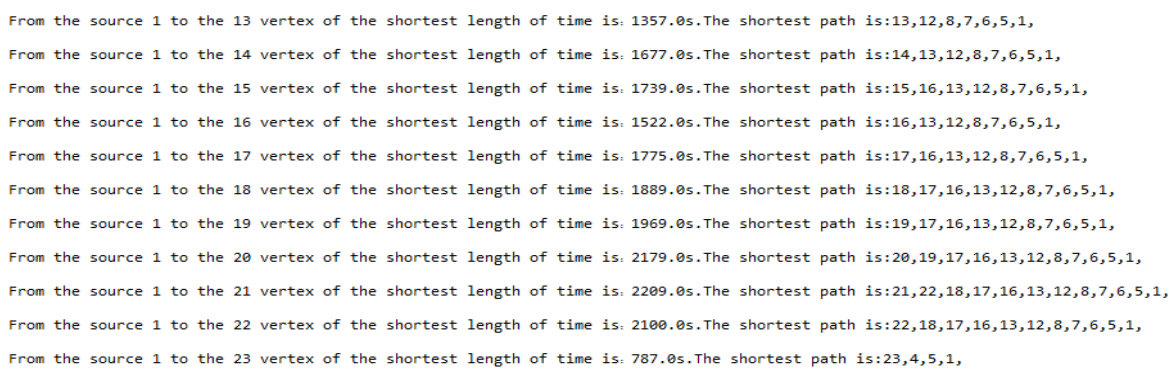

According to the above results, it can be very get from the source point 1 to the remaining 22 nodes of the shortest path length and the corresponding shortest time. Based on certain nodes around the building found from the provincial government, attractions to this purpose by the shortest path length and the corresponding shortest time.

\section{Example}

If someone get off at the provincial government (A) ICONS after the first target sites is small west lake (I) icon, can treat as is to node 15, in this way, the source point of the picture is the node 1 , the target node is 15.In the following two cases to analyze the problem:

(1) Problem can be solved by one of the shortest path, the best route for:

$$
\text { 1->5->6->7->9->10->14->15 }
$$

The route a total length of 2450 meters, the length of time of 612.5 seconds.

(2) With the length of the shortest time to solve in the problem, the best route for:

$$
\text { 1->5->6-> 7->8->12->13->16->15 }
$$

The route a total length of 2941 meters, the length of time of 1739.0 seconds.

By visible, use the shortest path to consider not only the problem than the total length of the shortest route, the total length of time the desires of the length of the route and length of time than the shortest length of time length is much smaller, so the one-day tour plan in Lanzhou only with reference to the shortest path method to choose the path, and don't have to worry about the time.

\section{Evaluation of the model}

\section{The advantages of the model}

1) The shortest time and the shortest path problem, is included in the model can be a matter of time;

2) Application portability strong, according to the need to increase of arbitrary nodes, you can add a node and the corresponding code with arbitrary distance, so as to formulate the corresponding shortest path planning, saving travel time;

3) Model is practical, and the popularizing this model, offers visitors the best 
travel plan.

\section{The disadvantage of the model}

1) Covers an area of the larger overpass in the figure, only as a node may cause error;

2) The number of spots statistical model is less, should be how to add more attractions design.

\section{Acknowledgments}

This work was supported by the National Science Foundation of China (No.11401473), Natural Science

Foundation of Gansu Province(NO.145RJZA214), 2013 the basic scientific research in colleges and universities were a special fund project(NO.31920130006), Fundamental Research Funds for the Central Universities(NO.31920140058), Talent Introduction Scientific Research Foundati on of Northwest Universityfor Nationalities(NO.xbmuyjrc201305).

\section{Reference}

[1] W. Xiaodong, Algorithm design and analysis,edited by Beijing, Tsinghua university press, (2008).

[2] T.haoqiang,L.zunchao,S.Jun, The Java programming language, edited by Beijing(second edition), China railway press, (2007).

[3] D. Zaijun, Operations research(third edition), edited by Beijing, Higher education press , (2012).

[4] H. Yunquan, Operational research foundation and application (third edition), edited by Harbin, Harbin industrial university press, (1998).

[5]K. Man, Introduction to algorithms(third edition), edited by Beijing, Mechanical industry press, (2013)

[6] W. Qin, X.Lifeng, Data structures and algorithms, edited by Beijing, Tsinghua university press, (2012). 